\title{
Ultrasonic flaw detection of discontinuous defects in magnesium alloy materials
}

\author{
Sheng-nan Xue, *Qi-chi Le, Yong-hui Jia, Li-ping Jiang, Zhi-qiang Zhang, Lei Bao \\ Key Lab of Electromagnetic Processing of Materials, Ministry of Education, Northeastern University, Shenyang 110819, China
}

\begin{abstract}
Phased array ultrasonic testing, an effective ultrasonic testing (UT) technology, has been widely used in steel inspection because of its high accuracy, sensitivity, and efficiency. However, as its application in as-cast magnesium alloys has just begun, more research is needed. Considering the important role of the gain compensation in quantifying defects in magnesium alloys by ultrasonic phased array technology, the effects of microstructure, the position, size, and overlap of defects, and boundary distance (distance from the defect position to the side surface of the test casting) on gain compensation of as-cast AZ80 and AZ31 magnesium alloys were studied. Results show the gain compensation increases with the increase of grain size. There is a strict linear positive correlation between gain compensation and defect depth, but such relationship no longer exists due to the defects overlap, orientation and boundary distance. In addition, there is a strict linear negative correlation between the gain compensation and defect size.
\end{abstract}

Key words: magnesium alloy; ultrasonic phased array; defect detection; gain compensation

CLC numbers: TG115.285 Document code:A Article ID: 1672-6421(2019)04-256-06

$\mathrm{M}$ agnesium alloys are used as metal structural materials in the automotive and aerospace industries due to low density and easy recycling, and as bio-implant materials because of their high specific strength, perfect biocompatibility and natural abundance. Magnesium-aluminum-zinc alloy is one of the earliest and most widely used magnesium alloys, among which AZ31 and AZ80 magnesium alloys have high strength, plasticity and corrosion resistance. However, it has been found that cast magnesium alloys have serious problems of discontinuous defects due to the oxidation, combustion, and purification difficulty of the melt. Defect detection has become an important issue in the production and application of magnesium alloys ${ }^{[1,2,3]}$. Phased array ultrasonic testing, as an effective technology of Ultrasonic Testing (UT), has been widely used in steel and weld inspection because of its high accuracy, sensitivity, and efficiency ${ }^{[4,5]}$. However, as its application in ascast magnesium alloys has just begun, more research is needed.

The gain compensation is a key parameter for quantifying defects in ultrasonic phased array detection ${ }^{[6]}$. When the selected gain compensation is too great, the

\section{*Qi-zhi Le}

Male, born in 1968, Ph.D., Professor. His research interest focuses on the casting of magnesium alloys.

E-mail: qichil@mail.neu.edu.cn

Received: 2019-03-25; Accepted: 2019-06-10 bottom wave will interfere with the detection result and cause the defect to be misjudged. At the same time, the width of the reflection wave at both ends of the test sample becomes too large, resulting that the defect near the end surface cannot be accurately displayed. However, if the selected gain compensation is too small, defects of smaller size will be missed. Therefore, it is necessary to select appropriate gain compensation when detecting defects using an ultrasonic testing method.

Researchers had found that the gain compensation of defect detection in AZ91 magnesium alloy has a certain relationship with the diameter of hole defect and depth ${ }^{[7]}$. However, the quantity of defect studied is insufficient and the effect of boundary distance (distance from the defect position to the side surface of the test casting), defect orientation, and defect overlap on detection results is not taken into account; it has little guiding significance for the defect detection of magnesium alloys in the actual production process. Hence, we took as-cast AZ80 and AZ31 alloys, which are most widely used, as the research object, aimed at finding the effect of microstructure, the position and size of defects, and boundary distance on the gain compensating, obtaining an effective range of gain compensation to more accurately characterize defects. What's more, this study also lays a foundation for accurate defect quantification and provides a reference for further qualitative research of defects. 


\section{Experimental materials and procedures}

\subsection{Experimental materials and testing equipment}

In this experiment, the as-cast AZ80 and AZ31 magnesium alloy test blocks were studied. Three AZ80 test blocks were taken from a low-frequency electromagnetic casting ingot, and one AZ31 test block was taken from a semi-continuous casting slab. The sampling positions are shown in Fig. 1. The magnesium alloy materials used in this experiment were all ultrasonically detected by straight probes to ensure that there were no defect signals larger than the equivalent of $1 \mathrm{~mm}$ flat bottomed holes. Surfaces of all test blocks were polished to ensure full contact of the probe and the test block, reducing the effect of surface roughness on the test results ${ }^{[8]}$. The OmniScan MX portable module phased array detector was used, with a probe model of 5L64-A2 which consisted of 64 wafers in a one-dimensional linear arrangement with 16 wafers as a whole, with an operating frequency of $5 \mathrm{MHz}$. The detector's wedge type was SA2-0L-IHC, its nominal refractive angle of the ultrasonic wave was $0^{\circ}$, the ultrasonic type was longitudinal wave, and the coupling agent was water ${ }^{[9-12]}$. (a)

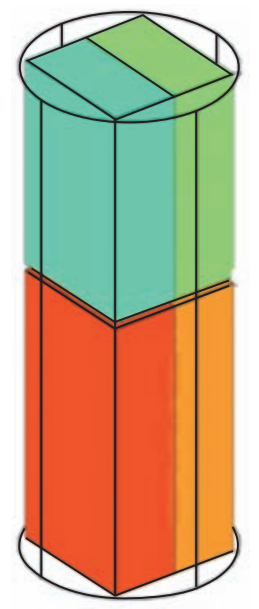

(b)

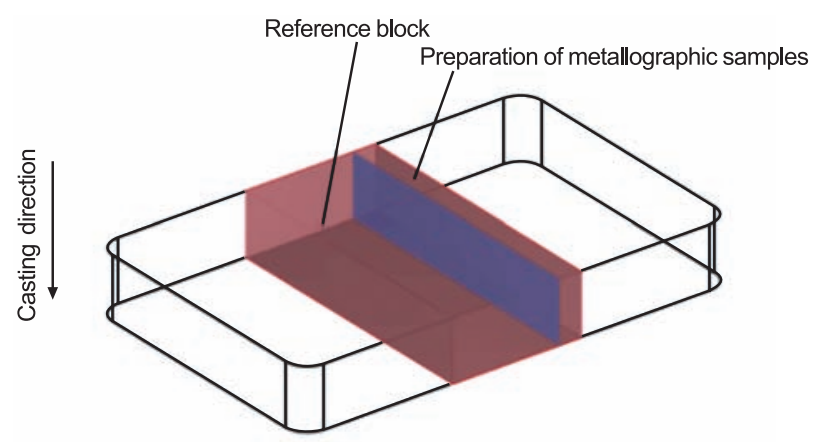

Fig. 1: Positions of test blocks: (a) low frequency electromagnetic casting ingot; (b) semi-continuous casting slab

\subsection{Experimental procedures}

Since it was difficult to collect a quantity of defect patterns for research in a short time, transverse holes were processed on different test blocks to replace defects in this experiment ${ }^{[13]}$. Test blocks were all $210 \mathrm{~mm} \times 100 \mathrm{~mm} \times 50 \mathrm{~mm}$ after machining. The three as-cast AZ80 test blocks were named AZ80- $\Phi 1,-\Phi 2$, - $\Phi 3$ according to the defect diameter of 1,2 , and $3 \mathrm{~mm}$. Ascast AZ31 test block had a defect diameter of $2 \mathrm{~mm}$. Defect distribution of different test blocks was the same, as shown in Fig. 2. In general, the probe emits a sound beam in the horizontal plane along the axial direction of the artificial hole, but when studying the effect of defect orientation on the gain compensation, the probe emits a sound beam in the vertical plane along the axial direction of the artificial hole ${ }^{[14]}$. The phased array transducer generated an ultrasonic signal with a frequency of $5 \mathrm{MHz}$ and a sound velocity of $5,800 \mathrm{~m} \cdot \mathrm{s}^{-1}$ under the excitation of high frequency electric signal which was transmitted to the phased array polycrystalline probe through the control system, and passed through the coupling medium to enter the interior of the sample to realize the ultrasonic wave sound field control. During the test, water was used as the coupling agent, the depth of focus was set to the thickness of the test block in the direction of propagation of the ultrasonic waves, the probe position was adjusted until its center faced the artificial hole which is located at different depths, and then a constant pressure was applied to the probe to ensure coupling.

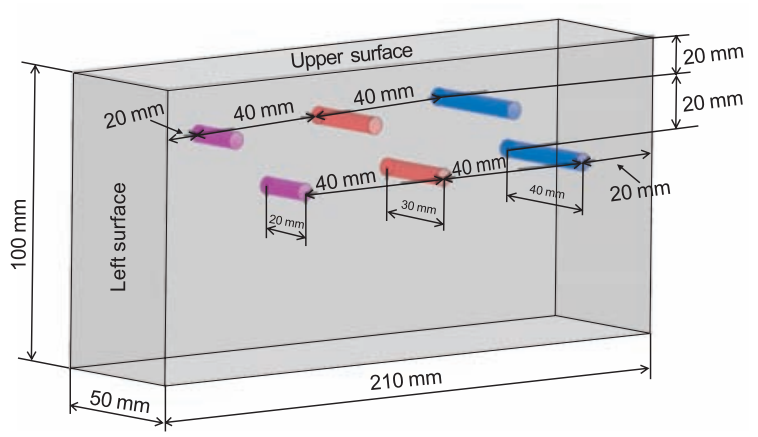

Fig. 2: Positions of defects

Under the premise that the center position of the probe aperture remained unchanged, the gain compensation $G$ was recorded when the echo at different depths $(H)$ reached $80 \%$ of the full screen height. To eliminate random interference, multiple measurements were taken for each point, and the average value was used.

\section{Results and discussion}

\subsection{Effect of defect position and grain size on gain compensation value}

The microstructure of the experimental alloy is shown in Fig. 3. The microstructure of the as-cast magnesium alloy is coarse dendrites with a great number of intergranular 


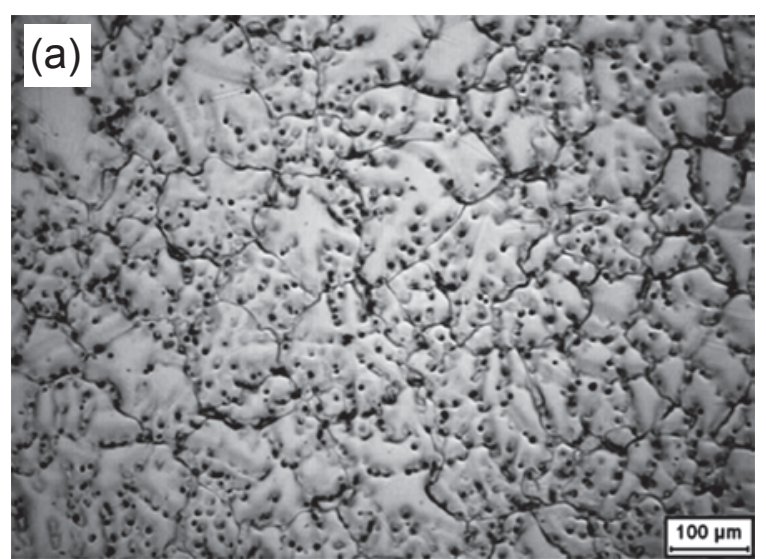

Fig. 3: Microstructure of as-cast AZ31 (a) and AZ80 (b) magnesium alloys

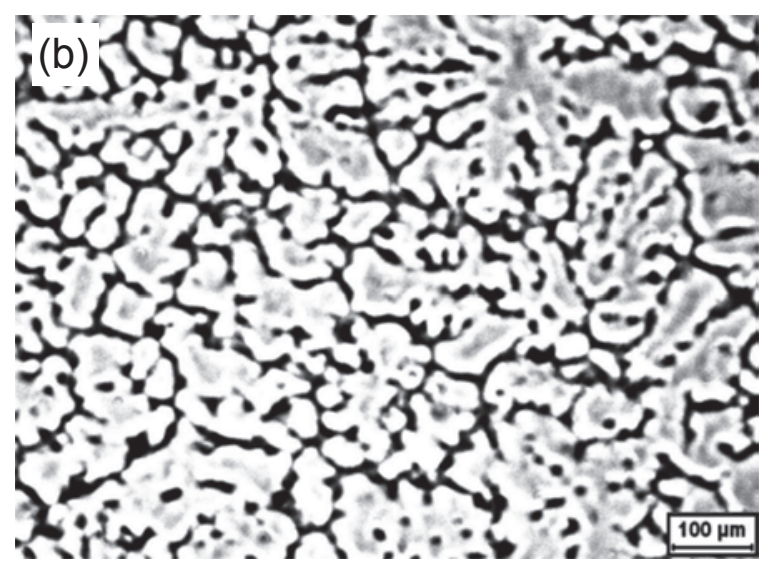

precipitation phases. The average grain size of AZ31 and AZ80 were $200 \mu \mathrm{m}$ and $300 \mu \mathrm{m}$, respectively.

Defects were detected on the surface $(210 \mathrm{~mm} \times 50 \mathrm{~mm})$ of as-cast AZ80- $\Phi 2$ and AZ31 blocks. During the test, the depth of focus was set to $100 \mathrm{~mm}$, and pressure was applied to ensure that the probe is in complete contact with the test block. The relationship between the measured gain compensation and the defect position is shown in Fig. 4.

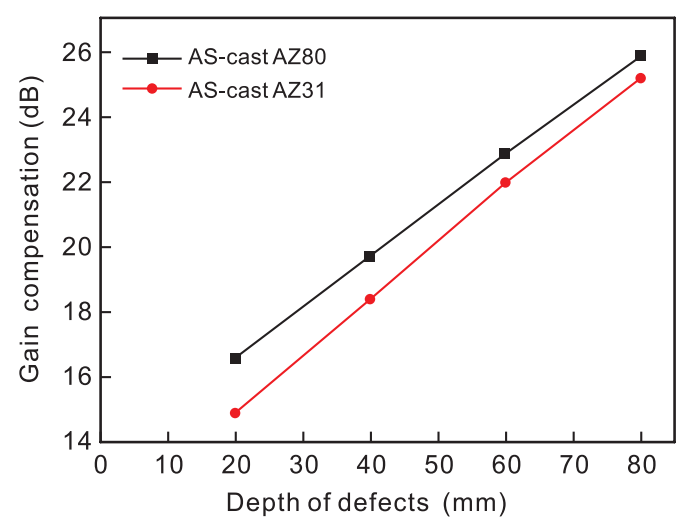

Fig. 4: Effect of defect position on gain compensation

Equations (1) through (2) are linearly regressed from Fig. 4.

$$
\begin{aligned}
& G=13.6+0.154 H \\
& G=11.5+0.173 H
\end{aligned}
$$

where $H$ is the distance from the detected surface to the center of the defect, $G$ is gain compensation. Equations (1) and (2) show the relationship between defect position and gain compensation of as-cast AZ80 and AZ31. It can be seen that the gain compensation is linearly correlated with the defect position, that is $G=k_{1} \cdot D+b_{1}\left(k_{1}>0\right)$, where $b_{1}$ is intrinsic compensating value reflecting the material types; $k_{1}$ is the index reflecting the material's grain size and orientation. As the depth of the defect increased, the gain compensation of the system gradually increased. However, the results of defects of the same depth and diameter are different in different test blocks, which mean that the ultrasonic waves have different attenuation degrees when they propagate in different compositions of magnesium alloys. For as-cast magnesium alloys, the gain compensation required for AZ80 alloy to detect defects is 1-3 dB larger than AZ31 alloy (Fig. 4). The reason is that the grain size is positively correlated with the gain compensation required for defect detection. The larger the grain size, the more severe the attenuation of ultrasonic wave needed during propagation, and the greater the gain compensation required for defect detection.

\subsection{Effect of defect size on gain compensation value}

The experiment was performed on the test surface $(210 \mathrm{~mm}$ $\times 50 \mathrm{~mm}$ ) of the as-cast AZ80- $\Phi 1$, AZ80- $\Phi 2$, and AZ80- $\Phi 3$ blocks. During the test, the depth of focus was set to $100 \mathrm{~mm}$, and pressure was applied to ensure the coupling of probe and test block. The relationship between the measured gain compensation and the defect size is shown in Fig. 5.

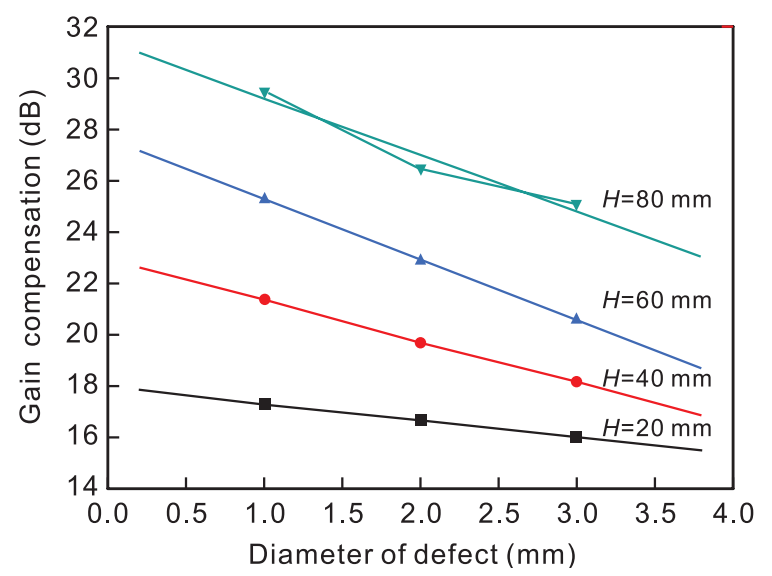

Fig. 5: Relationship between gain compensation and diameter of defects

Equations (3) through (6) are linearly regressed from Fig. 5.

$$
\begin{aligned}
& G=18.0-0.65 D, H=20 \mathrm{~mm} \\
& G=23.0-1.60 D, H=40 \mathrm{~mm} \\
& G=27.6-2.35 D, H=60 \mathrm{~mm} \\
& G=31.4-2.40 D, H=80 \mathrm{~mm}
\end{aligned}
$$

where $D$ is defect diameter, $G$ is gain compensation. It can be 
noted that the gain compensation required for defect detection at different depths decreases with the increase of defect size, as the equation is: $G=k_{2} \cdot D+b_{2}\left(k_{2}<0\right)$, where $b_{2}$ is the feature compensation representing the depth of the defect; $k_{2}$ is a sensitivity index representing the required gain compensation as to different size of defects. $k_{2}$ becomes larger as the defect lay further from the detection surface. That is, the farther the defect is from the detection surface, the more drastic the change of the gain compensation with the defect diameter. Moreover, when the thickness of the tested material is greater, different gain compensation values should be selected to test defects of different diameters to avoid the defect being undetected.

\subsection{Effect of boundary distance on gain compensation value}

During the experiment, it was found that although the defects with the same diameter were at same depth, the measured gain compensation was different due to the influence of the distance from the defect to the side surface, where the boundary represents the left and right surfaces of the test blocks in this experiment, as shown in Fig. 2. Defects detection was carried on the upper and lower surfaces. Figure 6 shows the variation of the gain compensation affected by the boundary.

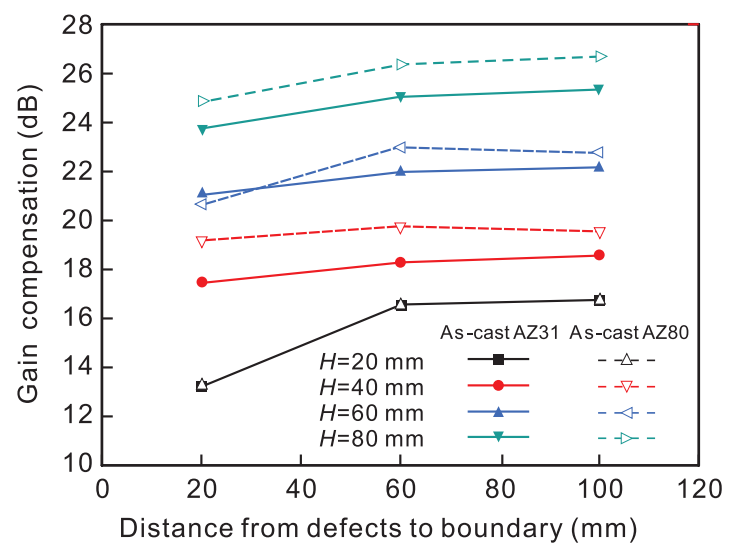

Fig. 6: Effect of distance from defect to boundary on gain compensation

It can be seen that the defects with boundary distance greater than $60 \mathrm{~mm}$ have similar detection results, but when that is less than $60 \mathrm{~mm}$, the gain compensation decreases with the decrease of the distance of defect from the boundary. Although the grain orientations and dimensions of different defects at the same depth are similar, the gain compensation required for detection is different, indicating that the boundary effect will affect detection results. During the propagation of the ultrasonic wave, the reflected wave is formed due to the grain boundary. When the wave encounters the boundary of the block, it will be re-reflected, and the reflected sound pressure of the wave gets larger, that is, the existence of the boundary reduces the scattering attenuation coefficient, so the defect can be judged when the system compensates for less energy ${ }^{[15]}$. Consequently, in the actual detection, in order to eliminate the influence of the boundary effect, the gain compensation should be appropriately reduced by $1-3 \mathrm{~dB}$. It can also be summarized that the trend of curves of different test blocks is almost the same, so the change of the gain compensation due to the boundary distance is not much different between the magnesium alloy test blocks of different compositions.

\subsection{Effect of defect overlap effect on gain compensation value}

Usually, a defect does not stand alone in actual alloys. When ultrasonic testing is performed, the reflected waves of different defects may interact with each other, making the detection result different from that of individual defects. Therefore, it is necessary to explore the variation law of the gain compensation when the defects are superimposed. Defects were detected on the $100 \mathrm{~mm} \times 50 \mathrm{~mm}$ detection surface, and the left and right surface of each test block were detected separately. The depth of focus was set to $100 \mathrm{~mm}$, and water was used as a coupling agent. The average was obtained after multiple measurements. The results are shown in Fig. 7.

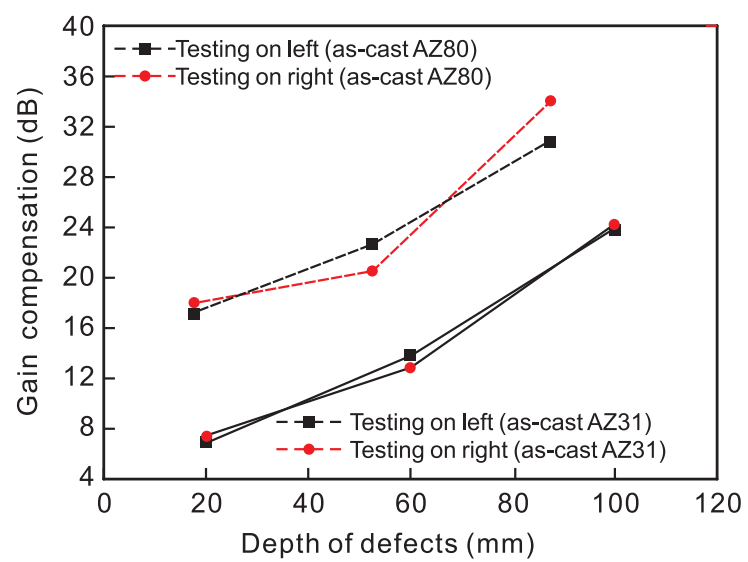

Fig. 7: Effect of defects overlap on gain compensation

The results in Section 2.1 show that the gain compensation is linearly dependent on defect depth. However, when defects are concentrated or superimposed upon each other, they are nonlinear. This is due to the interaction of reflected waves of different defects. Therefore, in the actual detection, multiple detection surfaces should be selected to avoid defect overlap to ensure the accuracy of the detection. It can be seen that the trend of the influence of the overlap of defects on different test blocks and test surfaces is different (Fig. 8), further demonstrating that the metallographic structure has an influence on test results. The coarser the grain, the greater of a gain compensation is needed.

\subsection{Effect of defect orientation on gain compensation value}

In order to investigate the effect of the defect orientation on the detection result, the artificial hole is detected on the surface $(210 \mathrm{~mm} \times 100 \mathrm{~mm})$. The defect orientation is orthogonal to the detection surface, which is equivalent to a point defect. During the test, the depth of focus was set to $50 \mathrm{~mm}$, pressure was applied to ensure coupling, and the average value was taken after multiple measurements. The results are shown in Fig. 8. 

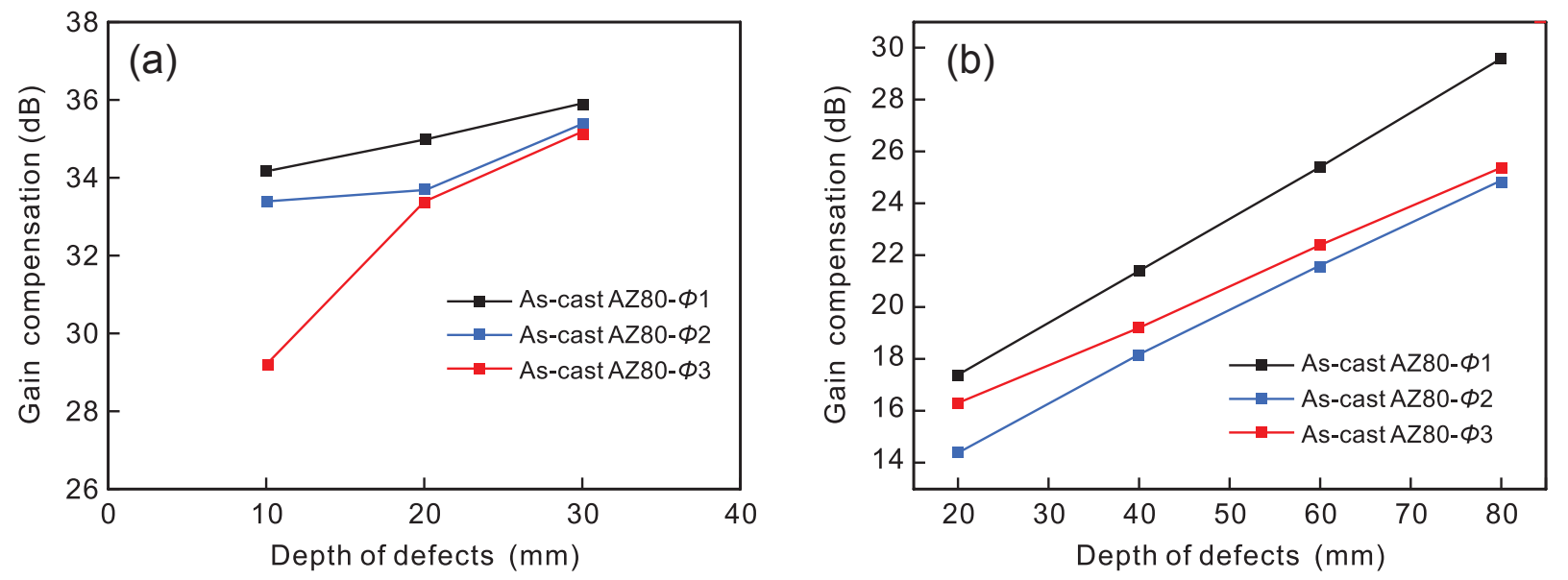

Fig. 8: Effect of defect orientation on gain compensation: (a) orthogonal; (b) parallel

From the above results, it can be concluded that the detection results of perpendicular to the defect orientation and that of parallel to the defect orientation are greatly different. The reason is that the longitudinal wave flaw detection mainly finds a defect which is parallel or slightly inclined to the detection surface, while the transverse wave flaw detection mainly finds a defect which is perpendicular to the detection surface or largely inclined to the detection surface, showing that defects of different orientations may be missed when using a single waveform for detection ${ }^{[16,17,18]}$. The defects inside the workpiece, its size, shape, depth, inclination angle, surface roughness, internal filling, and amount are unknown ${ }^{[19,20]}$. For the sake of locating and quantifying the defects accurately, it is necessary to measure it in different test surfaces. If the detected workpiece can only provide one detection surface, it is essential to perform longitudinal, and also transversal wave scanning to ensure the precision of the detection results.

\section{Conclusions}

The influences of the microstructure of AZ80 and AZ31 magnesium alloy and their defect position, defect size, defect overlap, and boundary distance on the gain compensation of ultrasonic phased array inspection were studied. The important conclusions can be drawn as follows:

(1) For the detection of different artificial holes in different magnesium alloys, the gain compensation obtained is positively linearly related to defect depth. Additionally, the coarser the grain, the larger of a gain compensation is required for detection.

(2) The gain compensation has a negative linear correlation with the defect diameter. The difference in defect size results in a larger change in gain compensation. Hence, when the thickness of a tested material is large, different gain values should be selected to test defects to avoid a defect being undetected.

(3) When it comes to the detection of defects near to the free surface of object with the distance less than $60 \mathrm{~mm}$, the boundary lays a serious influence on the gain compensation. In an actual test, the gain compensation should be decreased by
1-3 dB, taking the boundary effect into consideration.

(4) While the overlap of defects occurs, the accuracy of detection will be distracted by defects nearby. Defect orientation affects the test results as well. In order to accurately locate and distinguish defects, it is necessary to detect them in multiple different tests surface, or to perform both longitudinal and transversal wave scanning.

\section{References}

[1] Yu Junying. The Application of Ultrasound in the Flaw Detection of Mg-alloy Cast-ingots. Shanghai Metals, 1992: 26-31. (In Chinese)

[2] Froes $\mathrm{F} \mathrm{H}$, Eliezer D, Aghion E. The science, technology, and applications of magnesium. JOM, 1998, 50(9): 30-34.

[3] Ling Hao, Cheng B R. Sealing processes of anodic coatings Past, present, and future. Metal Finishing, 2000, 98(12): 8-18.

[4] Kuang Yinghui. Research on Modeling and Parameter Optimization of Ultrasonic Phased Array. Chinese Journal of Sensors \& Actuators, 2010, 23(12): 1731-1735.

[5] Li Zhongsheng, Pan Fusheng, Zhang Jing. Present Research Status and Development Prospect of AZ31 Magnesium Alloy. Metal Forming Technology, 2004: 54-72. (In Chinese)

[6] Shao Changlu, Han Zhongchao, Jiang Hao, et al. Water Immersion Ultrasonic Testing of Macroscopic Inclusions in Steel. Nondestructive Testing, 2018, 40(1): 75-77. (In Chinese)

[7] Zhao Mingshu, Chen Zhenhua, Shi Yaowu. Study on Ultrasonic Testing of Defects for AZ91 Cast Magnesium Alloy. Nondestructive Testing, 2008: 73-80. (In Chinese)

[8] Wu Wei. Experimental study on ultrasonic testing of tungsten and molybdenum alloys. World Nonferrous Metals, 2018: 270271. (In Chinese)

[9] Huang Min, Li Gong. Intelligent defect recognition methods in the ultrasonic non-destructive test of welds. Journal of Beijing Information Science \& Technology University, 2009, 24(2): 3336. (In Chinese)

[10] Jiao Jingpin, Li Yong, He Cunfu, et al. Study on Acoustic Emission Pipeline Leak Detection. Journal of Beijing Polytechnic University, 2003, 29(2): (144-146). (In Chinese)

[11] Cao Yunfeng, Hua Xiyang, Tian Weijian, et al. The Ultrasonic Testing Typical Case of Nickel-Based Alloy Bolt. Nondestructive Testing, 2016, 79-82. (In Chinese)

[12] Hanstock R F, Lumb R F, Walker D C B. Ultrasonic inspection of tubes. Ultrasonics, 1964, 2(3): 109-119. 
[13] Park J S, Song S J, Kim H J. Calculation of Radiation Beam Field from Phased Array Ultrasonic Transducers Using Expanded Multi-Gaussian Beam Model. Solid State Phenomena, 2006, 110: 163-168.

[14] Markov A A, Mosyagin V V, Keskinov M V. A program for 3D simulation of signals for ultrasonic testing of specimens. Russian Journal of Nondestructive Testing, 2005, 41(12): 778-789.

[15] Zhao Junru, Dai Guang, Zhang Ying. Experimental Research of the Acoustic Emission (AE) Characteristics during the Tensile Process of the Blowout Preventer Shell Material. Advanced Materials Research, 2012, 524-527(1): 1395-1398.

[16] Dahle A K, Sannes S, John D H S, et al. Formation of defect bands in high pressure die cast magnesium alloys. Journal of Light Metals, 2001, 1(2): 99-103.
[17] Zhu B, Cai X, Wang C G, et al. Studies on the relationship between acoustic emission characteristics and fracture toughness of materials. Acta Physica Sinica, 2003, 52(8): 1960-1964.

[18] Cáceres C H, Selling B I. Casting defects and the tensile properties of an Al Si Mg alloy. Materials Science \& Engineering A, 1996, 220(1-2):109-116.

[19] Crawford S. Assessment of Crack Detection in Heavy-Walled Cast Stainless Steel Piping Welds Using Advanced LowFrequency Ultrasonic Methods. Nondestructive Testing, December 2008, doi: 10.2172/921260.

[20] Kappes W, Rockstroh B, W Bähr, et al. Application of new front-end electronics for non-destructive testing of railroad wheel sets. Insight, 2007, 49(6): 345-349.

This work was financially supported by the National Key Research and Development Program of China (Grant No. 2017YFB0305504) and the National Natural Science Foundation of China (Grant No. 51771043). 